\title{
Agnostically learning under permutation invariant distributions
}

\author{
Karl Wimmer \\ Duquesne University \\ wimmerk@duq. edu
}

September 22, 2013

\begin{abstract}
We generalize algorithms from computational learning theory that are successful under the uniform distribution on the Boolean hypercube $\{0,1\}^{n}$ to algorithms successful on permutation invariant distributions, distributions where the probability mass remains constant upon permutations in the instances. While the tools in our generalization mimic those used for the Boolean hypercube, the fact that permutation invariant distributions are not product distributions presents a significant obstacle.

Under the uniform distribution, halfspaces can be agnostically learned in polynomial time for constant $\epsilon$. The main tools used are a theorem of Peres [Per04] bounding the noise sensitivity of a halfspace, a result of [KOS04] that this theorem implies Fourier concentration, and a modification of the Low-Degree algorithm of Linial, Mansour, and Nisan [LMN93] made by Kalai et. al. [KKMS08]. These results are extended to arbitrary product distributions in [BOW10].

We prove analogous results for permutation invariant distributions; more generally, we work in the domain of the symmetric group. We define noise sensitivity in this setting, and show that noise sensitivity has a nice combinatorial interpretation in terms of Young tableaux. The main technical innovations involve techniques from the representation theory of the symmetric group, especially the combinatorics of Young tableaux. We show that low noise sensitivity implies concentration on "simple" components of the Fourier spectrum, and that this fact will allow us to agnostically learn halfspaces under permutation invariant distributions to constant accuracy in roughly the same time as in the uniform distribution over the Boolean hypercube case.
\end{abstract}

\section{Introduction}

In this paper, we:

- Generalize the Low-Degree algorithm (and the agnostic learning algorithm of [KKMS08]) to the symmetric group, taking special care to account for the fact that the Fourier spectrum consists of matrices.

- Generalize the concept of noise sensitivity for a function $f: S_{n} \rightarrow \mathbb{R}$, and give an expression for noise sensitivity in terms of the Fourier spectrum of such functions.

- Prove that the noise sensitivity of generalized linear threshold functions $f: S_{N} \rightarrow \mathbb{R}$ have bounded noise sensitivity, and that this theorem will allow us to use the algorithm of [KKMS08] to agnostically learn these functions.

Our primary motivation is the class of binary classification problems over the instance space $\mathcal{X}=X^{n}$ for some set $X$ with $|X|=\operatorname{poly}(n)$. Consider the following algorithm for learning in such a scenario:

SVM box

We note that the above algorithm, which we will refer to as polynomial regression (as in [BOW10]), is a version of the wildly popular SVM algorithm. It is known that the above algorithm runs in poly $\left(m, n^{d}\right)$ with $m$ training examples. If $m=\Omega\left(n^{d} / \epsilon\right)$, the SVM algorithm is guaranteed (with high probability) to 
generalize to unseen data in the case that $\mathcal{X}$ is a product distribution [BOW10]. In fact, in [BOW10], the domain $\mathcal{X}$ is allowed to be the product of different sets, which can be of any finite cardinality (and even uncountably infinite under distributional assumptions). Further, to achieve any provable guarantee that the hypothesis generalizes, $\Theta\left(n^{d} / \epsilon\right)$ examples are necessary.

In the literature, much effort has been put into the case where the attributes are mutually independent. In this case, the data is drawn i.i.d. from a product distribution over $\mathcal{X}$. The uniform distribution over $\{0,1\}^{n}$ has received much attention in a number of different scenarios; some of these results extend to product distributions. This was the explicit motivation of [BOW10]. In [KST09], the authors show that learning of some natural concepts is possible under a randomly chosen product distribution with high probability. In practice, the assumption that the attributes are all mutually independent is unrealistic.

The assumption we make in this work is that the distribution is permutation invariant. By this, we mean that $\operatorname{Pr}_{\boldsymbol{X}}\left[\boldsymbol{X}=\left(\boldsymbol{x}_{1}, \boldsymbol{x}_{2}, \ldots, \boldsymbol{x}_{n}\right)\right]=\operatorname{Pr}_{\boldsymbol{X}}\left[\boldsymbol{X}=\left(\boldsymbol{x}_{\sigma}(1), \boldsymbol{x}_{\sigma}(2), \ldots, \boldsymbol{x}_{\sigma}(n)\right)\right]$ for any permutation $\sigma$. A permutation invariant distribution need not even be pairwise independent, although product distributions with equal biases for each coordinate are permutation invariant. Considering this type of distribution has already proven helpful in other work. In the context of learning monotone functions over the uniform distribution on $\{0,1\}^{n}$, [OW09] show that product distributions are too spread out for a natural approach to work. Converting to permutation invariant distributions of the previously mentioned form allows for stronger analysis. The approach in [OW09] is to learn monotone functions over one level of the Boolean cube at a time, focusing on each collection of examples with a fixed number of 1's.

To prove our results, we use tools from representation theory over the symmetric group. We define noise sensitivity for the symmetric group, and achieve similar learning results to those over the uniform distribution. The results and connections between computational learning theory and representation theory are interesting, and the representation theory results we show might be of independent interest.

\subsection{The learning framework}

Our goal is binary classification learning in the "agnostic" model introduced in [KSS94]. In this model, there is an unknown target function $t: \mathcal{X} \rightarrow\{-1,1\}$ which we are trying to recover. Our access to $t$ is limited; we receive labeled examples of the form $\langle x, t(x)\rangle$, where the marginal distribution on the first coordinate is some known distribution $\mathcal{D}$ on the set $\mathcal{X}$. Further, the examples are generated independently. The algorithm's task is to output a hypothesis $h: \mathcal{X} \rightarrow\{-1,1\}$ with minimal classification error with respect to $t$; that is, we wish to choose $h$ minimizing $\operatorname{err}_{t}(h)=\mathbf{P r}_{\boldsymbol{x} \sim \mathcal{D}}[h(\boldsymbol{x}) \neq t(\boldsymbol{x})]$. We compare the error of our hypothesis to the minimum error achievable using a function from some fixed class $\mathcal{C}$ of functions from $\mathcal{X}$ to $\{-1,1\}$. We say that we can "agnostically learn with respect to $\mathcal{C}$ under the distribution $\mathcal{D}$ " if, for any target function $t$, any $\epsilon>0$, given a bounded number of labeled examples, our algorithm returns a hypothesis satisfying

$$
\mathbf{E}\left[\operatorname{err}_{t}(h)\right] \leq \inf _{f \in C} \operatorname{err}_{t}(f)+\epsilon
$$

where the expectation is over the randomness of our algorithm.

In light of strong computational hardness results for such problems when the distribution is arbitrary, much work has gone into the case where the distribution $\mathcal{D}$ is a product distribution; that is, a distribution where all of the coordinates are mutually independent. The advantage of such a distribution is the ease with which an orthogonal decomposition can be constructed and analyzed.

\section{Algorithms for learning}

We recount some algorithms for learning. First, we make a definition:

Definition 2.1. For a function $f:\{0,1\}^{n} \rightarrow\{-1,1\}$, we say that $f$ is $\epsilon$-concentrated to degree $d$ with respect to $\mathcal{D}$ if there exists a polynomial $p$ of degree at most d such that $\mathbf{E}_{\boldsymbol{x} \sim \mathcal{D}}\left[(f(\boldsymbol{x})-p(\boldsymbol{x}))^{2}\right] \leq \epsilon$. We say that a class of functions $\mathcal{C}$ is $\epsilon$-concentrated to degree $d$ if every $f \in \mathcal{C}$ is $\epsilon$-concentrated to degree $d$. 
The concept of $\epsilon$-concentration has proved fruitful in computational learning theory. The Low-Degree Algorithm of Linial, Mansour, and Nisan was the first result to use $\epsilon$-concentration. In their paper, the authors considered learning under the uniform distribution on $\{0,1\}^{n}$. They show that if the target function $t$ is computable by a size $s$, depth $c$ circuit, then $t$ is $\epsilon$-concentrated to degree $O(\log (s / \epsilon))^{c}$. We note that the original result does not hold in the agnostic framework; the assumption here is that $t \in \mathcal{C}$; specifically here, $\mathcal{C}$ is the class of functions computable by some size $s$, depth $c$ circuit.

While our results can be applied to learning circuits, our primary focus here is the class of linear threshold functions.

Definition 2.2. We say that a function $f:\{0,1\}^{n} \rightarrow\{-1,1\}$ is a linear threshold function if there exists weights $w_{1}, w_{2}, \ldots, w_{n}$ and a threshold $\theta$ such that $f(x)=\operatorname{sgn}\left(\sum_{i=1}^{n} w_{i} x_{i}-\theta\right)$ for all $x \in\{0,1\}^{n}$.

In [KOS04], Klivans, O'Donnell, and Servedio develop the "noise sensitivity method" along these lines. They show $\epsilon$-concentration results for functions with bounded noise sensitivity. Specifically, they show that any function $f:\{0,1\}^{n} \rightarrow\{-1,1\}$ that can be written as a function of $k$ linear threshold functions is $\epsilon$ concentrated to degree $O\left(k^{2} / \epsilon^{2}\right)$ under the uniform distribution on $\{0,1\}^{n}$. Again, the result in their paper is only applicable to learning when $t$ is in the concept class $\mathcal{C}$.

The work in [KKMS08] gives a significant step forward in this line of work. In their paper, they consider the agnostic problem, where we do not assume $t \in \mathcal{C}$. In that paper, the authors show that $L_{2}$-approximability bounds can be used to imply $L_{1}$-approximability bounds, which can be used to achieve results in the agnostic setting. Under the uniform distribution on $\{0,1\}^{n}$, they show that $\epsilon^{2}$-concentration up to degree $d$ implies agnostic learning with accuracy loss $\epsilon$. Their algorithm is slightly different than the Low-Degree algorithm; their algorithm solves a linear program for a degree- $d$ polynomial $p(x)$ that minimizes absolute $L_{1}$ error. They also note that this is a version of the Support Vector Machines (SVM) algorithm.

A further step in this direction is given in [BOW10]. Blais, O'Donnell, and Wimmer show that nearly all results about $\epsilon$-concentration with respect to the uniform distribution on $\{0,1\}^{n}$ can be applied to arbitrary product distributions; distributions where the coordinates are mutually independent, but each coordinate has an arbitrary distribution. The main tools therein are an extension of the noise sensitivity method of [KOS04] to product distributions and an application of the algorithm of [KKMS08]. In this paper, we use both of these techniques, suitably adjusted for functions over the symmetric group.

The main technique we will use comes from representation theory of the symmetric group. We recall the work of Boneh [Bon95], which used representation theory of groups of size $2^{n}$ to establish learning results for the uniform distribution on the hypercube. The symmetric group is not mentioned in Boneh's paper. We note that learning results of a more applied nature are known for the symmetric group, particularly in the realm of multi-object tracking [KB08],[KHJ07],[HGG09]. All of these results make heavy use of representation theory; this document combines the techniques used in these works with the tools and ideas from the field of computational learning theory. Further, [SJ06] introduces a similar idea which they call permutation-invariant SVMs, where the classifier is forced to be invariant under permutations. We note that our assumption is that the distribution is invariant under permutations, but the classifier need not be.

\section{Overview of results}

The main result of this paper is the following:

Theorem 3.1. Let $\mathcal{C}$ be the class of functions of $k$ linear threshold functions over $\{0,1\}^{n}$, and let $\mathcal{D}$ be any permutation invariant distribution over $\{0,1\}^{n}$. There is an algorithm that agnostically learns with respect to $\mathcal{C}$ under the distribution $\mathcal{D}$, using $n^{O\left(k^{2} / \epsilon^{4}\right)}$ time and examples.

Our main theorem is actually a corollary of a stronger result:

Theorem 3.2. Let $\mathcal{C}$ be the class of functions of $k$ linear threshold functions over $S_{n}$. There is an algorithm that agnostically learns $\mathcal{C}$ under the uniform distribution on $\mathcal{D}$, using $n^{O\left(k^{2} / \epsilon^{4}\right)}$ time and examples. 
Definition 3.3. We say that a function $f: S_{n} \rightarrow\{-1,1\}$ is a linear threshold function if there exist weights $w_{i j}$ for $1 \leq i \neq j \leq n$ and a threshold $\theta$ such that $f(\sigma)=\operatorname{sgn}\left(\sum_{i, j} w_{i j} \mathbf{1}[\sigma(i)=j]-\theta\right)$ for all $\sigma \in S_{n}$.

We will prove our main theorem as a corollary of Theorem 3.2, as well as the following stronger result:

Theorem 3.4. Let $\mathcal{C}$ be the class of functions of $k$ linear threshold functions over $\mathcal{X}=\{1,2, \ldots, B\}^{n}$, and let $\mathcal{D}$ be any permutation invariant distribution over $\mathcal{X}$. There is an algorithm that agnostically learns with respect to $\mathcal{C}$ under the distribution $\mathcal{D}$, using $n^{O\left(k^{2} / \epsilon^{4}+B\right)}$ time and examples.

We note that in a permutation invariant distribution, the attributes need not even be pairwise independent. The class of permutation invariant distributions includes some product distributions as a special case. In some sense, permutation invariant distributions are a generalization of $p$-biased distributions.

To prove the above theorems, we prove the following generalization of Peres' Theorem [Per04], for a proper definition of noise sensitivity:

Theorem 3.5. Let $f: S_{n} \rightarrow\{-1,1\}$ be a linear threshold function. Then $\mathbb{N S}_{\delta}(f) \leq O(\sqrt{\delta})$.

We note that the bound $O(\sqrt{\delta})$ is the same bound for product distribution versions of this theorem. The advancement of noise sensitivity bounds is very recent; the uniform distribution case is discussed in [Per04], and the arbitrary product distribution case is proved in [BOW10].

Most of our efforts will be put into learning functions where the domain in $S_{n}$; we reduce to other distributions by identifying letters of the permutation. For example, if we can learn under the uniform distribution over permutations of $(1,2,3, \ldots, n)$, it is intuitively plausible that we can learn under the uniform distribution on permutations of $1^{n / 2} 0^{n / 2}$ (here we mean the vector of $n / 21$ 's followed by $n / 20$ 's) by identifying $\{1,2,3, \ldots, n / 2\}$ as indistinguishable as well as $\{n / 2+1, n / 2+2, \ldots, n\}$. This is indeed the case for the classes of functions we consider. Using the same technique as seen in [BOW10] for learning under mixtures of distributions, we prove the main theorem.

\section{Preliminaries}

\subsection{Representation theory}

We begin with representation theory of general groups.

Definition 4.1. We say that a representation $\rho$ of a group $G$ is a mapping $\rho: G \rightarrow \mathbb{R}^{d_{\rho} \times d_{\rho}}$ which preserves the algebraic structure of $G$; that is, for $\sigma_{1}, \sigma_{2} \in G$ we have $\rho\left(\sigma_{1} \sigma_{2}\right)=\rho\left(\sigma_{1}\right) \cdot \rho\left(\sigma_{2}\right)$. The matrices in the codomain of $\rho$ are called the representation matrices, and $d_{\rho}$ is the degree of the representation.

Definition 4.2. The character $\chi_{\rho}$ of a representation $\rho$ is a mapping $\chi_{\rho}: G \rightarrow \mathbb{R}$ such that $\chi_{\rho}(\sigma)=\operatorname{tr} \rho(\sigma)$.

We will be concerned with representations that are irreducible. These are representations that can not be decomposed into simpler representations (for some suitable definition of decompose). We say that two representations $\rho_{1}$ and $\rho_{2}$ are equivalent if there exists a nonsingular matrix $C$ such that $\rho_{2}(\sigma)=C^{-1} \rho_{1}(\sigma) C$ for all $\sigma \in G$. A set of all possible irreducible representations such that no two are equivalent contains all information about the structure of $G$. We will not be concerned with any specific such set of irreducible representations, but we mention that there are certain canonical choices. It is well-known that if $G$ is abelian, then all the irreducible representations have degree 1.

The Peter-Weyl Theorem says that the functions given in the matrix entries of irreducible representations of $G$ form an orthogonal basis for $L^{2}(G)$. For any group $G$, we have the following definition.

Definition 4.3. Let $f: G \rightarrow \mathbb{R}$ be any function on $G$, and let $\rho$ be any representation on $G$. The Fourier coefficient of $f$ at the representation $\rho$ is given by the matrix ${ }^{1}$

\footnotetext{
${ }^{1}$ This definition is slightly nonstandard; the division by $|G|$ is usually omitted. We use this definition to retain similarity to the literature on the Boolean hypercube.
} 


$$
\widehat{f}_{\rho}=\frac{1}{|G|} \sum_{\sigma \in G} f(\sigma) \rho(\sigma)
$$

The collection of the matrices $\widehat{f}_{\rho}$ at irreducible representations of $G$ is called the Fourier transform of $f$.

We can reconstruct $f$ from its Fourier transform via the Fourier Inversion formula:

$$
f(\sigma)=\sum_{\rho} d_{\rho} \operatorname{tr}\left(\hat{f}_{\rho}^{T} \rho(\sigma)\right)
$$

The tr expression can be thought of as a dot product between two length- $d_{\rho}^{2}$ vector versions of $\widehat{f}_{\rho}$ and $\rho$, arranging each matrix into a vector by taking the elements first top to bottom, then left to right as vectors.

\subsection{Boolean hypercube}

In the Boolean hypercube case, we consider functions of the form $f: \mathbb{Z}_{2}^{n} \rightarrow\{-1,1\}$. We think of the group $\mathbb{Z}_{2}^{n}$ as generated by the $n$ standard basis vectors of the form $e^{i}=(0, \cdots, 0,1,0, \cdots, 0)$, where $\left(e^{i}\right)_{i}=1$. Since $\mathbb{Z}_{2}^{n}$ is abelian, all the representations have degree 1 , and it is common to identify representations and characters; we use the symbol $\chi$. There are $2^{n}$ inequivalent irreducible representations, which are in bijection with $\mathbb{Z}_{2}^{n}$ in a natural way. For $\alpha \in\{0,1\}^{n}$, we define $\chi_{\alpha}: \mathbb{Z}_{2}^{n} \rightarrow\{-1,1\}$ such that $\chi_{\alpha}(x)=(-1)^{\sum_{i=1}^{n} \alpha_{i} x_{i}}$ (multiplication can be done in the ring $\left.\mathbb{Z}_{2}^{n}\right)$. We consider the vector space $L^{2}\left(Z_{2}^{n}\right)$, and we equip this vector space with the inner product defined by $\langle f, g\rangle=\mathbf{E}_{\boldsymbol{x}}[f(\boldsymbol{x}) g(\boldsymbol{x})]$, where $\boldsymbol{x}$ has the uniform distribution. The functions $\left\{\chi_{\alpha}\right\}$ form an orthonormal basis for this vector space, and it follows that every function $f: \mathbb{Z}_{2}^{n} \rightarrow \mathbb{R}$ can be uniquely written $f=\sum_{\alpha \in\{0,1\}^{n}} \hat{f}(\alpha) \chi_{\alpha}$ for some constants $\hat{f}(\alpha)$. These constants are called the Fourier coefficients of $f$, and together make up the Fourier spectrum of $f$.

We have Parseval's identity, which implies that $\sum_{\alpha} \hat{f}(\alpha)^{2}=1$ for a function $f: \mathbb{Z}_{2}^{n} \rightarrow\{-1,1\}$. This is a special case of Plancherel's identity, which states that $\langle f, g\rangle=\mathbf{E}_{\boldsymbol{x}}[f(\boldsymbol{x}) g(\boldsymbol{x})]=\sum_{\alpha} f(\alpha) g(\alpha)$ for $f, g: \mathbb{Z}_{2}^{n} \rightarrow$ $\mathbb{R}$; Parseval follows by taking $g=f$ and noticing that $f^{2} \equiv 1$.

For a subset $S$ of $\{0,1\}^{n}$, we say that a function $f: \mathbb{Z}_{2}^{n} \rightarrow\{-1,1\}$ is $\epsilon$-concentrated on $S$ if $\sum_{\alpha \in S} \hat{f}(\alpha)^{2} \geq$ $1-\epsilon$. Let $f_{S}=\sum_{\alpha \in S} \hat{f}(\alpha) \chi_{\alpha}$. Then for $f: \mathbb{Z}_{2}^{n} \rightarrow \mathbb{R}$, we have $\mathbf{E}_{\boldsymbol{x}}\left[\left(f(\boldsymbol{x})-f_{S}(\boldsymbol{x})\right)^{2}\right] \leq \epsilon$. It is well-know that if $f: \mathbb{Z}_{2}^{n} \rightarrow\{-1,1\}$ is $\epsilon$-concentrated on $S$, then $\operatorname{Pr}_{\boldsymbol{x}}\left[f(\boldsymbol{x}) \neq \operatorname{sgn}\left(f_{S}(\boldsymbol{x})\right)\right] \leq \epsilon$.

Let $P_{d}=\left\{x \in\{0,1\}^{n}:|x| \leq d\right\}$. The span of the functions $\chi_{\alpha}$ where $\alpha \in P_{d}$ is the space of all degree- $d$ polynomials over $\mathbb{Z}_{2}^{n}$. In the case that $f$ is $\epsilon$-concentrated on $P_{d}$, we say that $f$ is $\epsilon$-concentrated to degree $d$, and we recover Definition 2.1.

We define the influence of a coordinate $i$ as $\operatorname{Inf}_{i}(f)=\operatorname{Pr}_{\boldsymbol{x}}\left[f(\boldsymbol{x}) \neq f\left(e^{i}+\boldsymbol{x}\right)\right]$, and the total influence as $\operatorname{Inf}(f)=\sum_{i=1}^{n} \operatorname{Inf}_{i}(f)$. For a set $S$, let $\boldsymbol{x}^{S}$ denote $\boldsymbol{x}$ with the coordinates of $S$ rerandomized. Then $\mathbb{N S}_{\delta}(f)=\operatorname{Pr}_{\boldsymbol{x}, \boldsymbol{S}}\left[f(\boldsymbol{x}) \neq f\left(\boldsymbol{x}^{\boldsymbol{S}}\right)\right]$, where $\boldsymbol{S}$ is a uniformly randomly chosen subset of $[n]$ with cardinality $\operatorname{Binomial}(\delta, n)$. Equivalently, we can draw $\boldsymbol{x}^{\boldsymbol{S}}$ by independently rerandomizing each coordinate of $\boldsymbol{x}$ with probability $\delta$; it is easy to see that $\boldsymbol{S}$ is simply the set of coordinates rereandomized.

These quantities have nice interpretations in terms of the Fourier coefficients of $f$ :

$$
\operatorname{Inf}_{i}(f)=\sum_{\alpha: \alpha_{i}=1} \hat{f}(\alpha)^{2} \quad \operatorname{Inf}(f)=\sum_{\alpha}|\alpha| \hat{f}(\alpha)^{2} \quad \mathbb{N S}_{\delta}(f)=\sum_{\alpha}\left(\frac{1}{2}-\frac{1}{2}(1-2 \delta)^{|\alpha|}\right) \hat{f}(\alpha)^{2}
$$

In the latter two expressions above, the coefficients of $\hat{f}(\alpha)^{2}$ increase as $|\alpha|$ increases, and it follows that if $\operatorname{Inf}(f)$ or $\mathbb{N S}_{\delta}(f)$ is small enough, then $f$ is $\epsilon$-concentrated to degree $d$ for sufficiently large $d$.

As an example, in [Per04], it is proved that if $f:\{0,1\}^{n} \rightarrow\{-1,1\}$ is a linear threshold function, then $\mathbb{N S}_{\delta}(f) \leq O(\sqrt{\delta})$. It follows from [KOS04] that any function $f:\{0,1\}^{n} \rightarrow\{-1,1\}$ with this noise sensitivity bound is $\epsilon$-concentrated on $P_{d}$ where $d=O\left(1 / \epsilon^{2}\right)$. 


\subsection{The symmetric group and Young tableaux}

In this section we consider $L^{2}\left(S_{n}\right)$, the class of functions $f: S_{n} \rightarrow \mathbb{R}$ as a vector space of dimension $n$ ! under standard addition and scalar multiplication operations. We equip this space with the inner product $\langle f, g\rangle=\mathbf{E}_{\boldsymbol{\sigma}}[f(\boldsymbol{\sigma}) g(\boldsymbol{\sigma})]$.

Since the symmetric group is not abelian, Fourier analysis becomes much more involved. We will be very interested in the irreducible representations of $S_{n}$. We define a partition $\lambda$ of $n$ to be a nonincreasing sequence of integers $\left(\lambda_{1}, \lambda_{2}, \ldots, \lambda_{m}\right)$, where $\sum_{i} \lambda_{i}=n$ and each $\lambda_{i}>0$. We write this as $\lambda \vdash n$. The following is well-known:

Theorem 4.4. The irreducible representations of $S_{n}$ are indexed by partitions of $n$.

It is common to use Ferrer's diagrams to visualize a partition of $n$. The Ferrer's diagrams represent each component of each partition as the number of squares in the corresponding row. A standard Young tableau is a Ferrer's diagram with the numbers $1,2, \ldots, n$ each occurring in the one cell, such that the numbers in the cells are increasing downwards and to the right. (We refer the reader to [Ful97] for a thorough treatment of Young tableaux.) We define the dominance order on partitions of $n$ in the following way: we write $\lambda \unrhd \mu$ if $\sum_{i=1}^{k} \lambda_{i} \geq \sum_{i=1}^{k} \mu_{i}$ for all $i$, where we pad the partitions with extra zeroes where necessary.

Another combinatorial concept that will be important for us is the concept of skew diagrams. Given partitions $\lambda \vdash n$ and $\mu \vdash k$, a skew diagram of shape $\lambda / \mu$ is the diagram formed by the set-theoretic difference of $\lambda$ and $\mu$. A skew-standard Young tableau is a skew diagram filled with 1 up to the number of cells in the diagram (which may be more than $n-k$ ), each number occurring in exactly one cell, where the numbers increase downwards and to the right. We will only be concerned with skew tableau with $\mu=(k)$. Further, we will say that there are no skew tableau of shape $\lambda / \mu$ if $\lambda_{1}<\mu_{1}$.

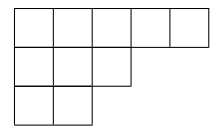

A standard diagram of shape $(5,3,2)$

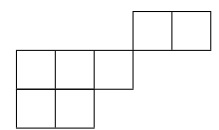

A skew diagram of shape $(5,3,2) /(3)$.

We say that the degree of a Ferrer's diagram of shape $\lambda$ is the number of standard Young tableaux of shape $\lambda$, and we denote the degree $\operatorname{dim} \lambda$ or $d_{\lambda}$. We will also refer to this as the degree of the partition $\lambda$. Similarly, we define $\operatorname{dim} \lambda / \mu$ as the number of skew-standard Young tableaux of shape $\lambda / \mu$. A variety of expressions for these dimensions are known.

Because of the equivalence of irreducible representations and partitions, we will frequently identify $\rho_{\lambda}$ and $\lambda$, where $\rho_{\lambda}$ is an irreducible representation corresponding to the class of representations indexed by the partition of $\lambda$. We will shorten $\rho_{\lambda}$ to $\rho$ when the correspondence is clear from context. The fact that we called the degree of a Ferrer's diagram $d_{\rho}$ is suggestive of the following theorem:

Theorem 4.5. The degree of any irreducible representation is equal to the degree of the Ferrer's diagram of the corresponding partition. That is, $d_{\lambda}=d_{\rho_{\lambda}}$, which we will write as $d_{\rho}$ when clear from context. Further, we can write $\widehat{f}_{\lambda}$ in place of $\widehat{f}_{\rho}$.

We define a simple function of degree $d$ to be a function $f: S_{n} \rightarrow \mathbb{R}$ such that there exists $d$ coordinates $1 \leq i_{1}<i_{2}<\ldots<i_{d} \leq n$ such that $f(\sigma)$ is completely determined by $\sigma\left(i_{1}\right), \sigma\left(i_{2}\right), \ldots, \sigma\left(i_{d}\right)$ for all $\sigma$. A function of degree $d$ is a function expressible as a linear combination of simple functions of degree $d$. Let $P_{d}^{\prime}=\left\{\lambda \vdash n: \lambda_{1} \geq n-d\right\}$. Then the span of the set of functions $\left\{\rho_{\lambda}: \lambda \in P_{d}^{\prime}\right\}$ is exactly the vector space of functions of degree $d$.

We have Parseval's identity, which says that for $f: S_{n} \rightarrow\{-1,1\}$, we have $\sum_{\lambda \vdash n} d_{\lambda}\left\|\hat{f}_{\lambda}\right\|^{2}=1$. All matrix norms are the Frobenius norm: $\|A\|=\sqrt{\operatorname{tr}\left(A^{T} A\right)}=\sqrt{\sum_{i, j} A_{i j}^{2}}$. Parseval's identity follows from Plancherel's identity: $\langle f, g\rangle=\sum_{\lambda \vdash n} d_{\lambda} \operatorname{tr}\left(\hat{f}_{\lambda}^{T} \hat{g}\right)$. 
We can now give the proper generalization of the Fourier interpretation of $\epsilon$-concentration. Let $S$ be a set of partitions of $n$. We say that $f: S_{n} \rightarrow \mathbb{R}$ is $\epsilon$-concentrated on a set $S$ if $\sum_{\lambda \in S} d_{\lambda}\left\|\hat{f}_{\lambda}\right\|^{2} \geq 1-\epsilon$. If $f$ is $\epsilon$-concentrated on $P_{d}^{\prime}$, then $f$ is $\epsilon$-concentrated to degree $d$, as in Definition 2.1.

As in previous work in the literature, we will show that functions $f: S_{n} \rightarrow\{-1,1\}$ with low noise sensitivity achieve $\epsilon$-concentration to degree $d$. However, the "right" definition of noise sensitivity is not immediate. From a random walk viewpoint, it is most natural to define noise sensitivity of a function $f: S_{n} \rightarrow\{-1,1\}$ as the probability that applying a random sequence of transpositions to an element changes its image under $f$. To more closely mimic the combinatorial interpretation of noise, we use a slightly different kind of noise sensitivity.

\section{$5 \quad$ Noise sensitivity}

For $0 \leq \delta \leq 1$, we define the distribution $N_{\delta}$ to be the distribution over $S_{n}$ whose samples can be generated as follows: select $\boldsymbol{k} \sim \operatorname{Binomial}(n, \delta)$, and a uniformly random subset $\boldsymbol{S} \subseteq[n]$ of cardinality $\boldsymbol{k}$. The sample is a uniformly random permutation such that every coordinate outside of $\boldsymbol{S}$ is a fixed point of the permutation.

Definition 5.1. We define the functional operator $T_{\delta}$ such that, given a function $f: S_{n} \rightarrow \mathbb{R}$, the function $T_{\delta} f$ is defined such that

$$
\left(T_{\delta} f\right)(\sigma)=\mathbf{E}_{\boldsymbol{\psi} \sim N_{\delta}}(\sigma)[f(\boldsymbol{\psi})]=\mathbf{E}_{\boldsymbol{\psi} \sim N_{\delta}}[f(\boldsymbol{\psi} \sigma)]=\mathbf{E}_{\boldsymbol{\psi} \sim N_{\delta}}[f(\sigma \boldsymbol{\psi})]
$$

The last equality above uses the fact the distribution on $N_{\delta}$ is a class distribution; $\operatorname{Pr}_{\psi \sim N_{\delta}}[\boldsymbol{\psi}=\tau]=$ $\operatorname{Pr}_{\psi \sim N_{\delta}}\left[\boldsymbol{\psi}=\sigma \tau \sigma^{-1}\right]$ for all $0 \leq \delta \leq 1$ and all $\tau, \sigma \in S_{n}$.

Definition 5.2. Given a function $f: S_{n} \rightarrow\{-1,1\}$, we denote the noise sensitivity of $f$ at $\delta$ as $\mathbb{N S}_{\delta}(f)$, and we define this quantity as

$$
\mathbb{N S}_{\delta}(f)=\operatorname{Pr}_{\boldsymbol{\sigma}, \boldsymbol{\psi} \sim N_{\delta}}[f(\boldsymbol{\sigma}) \neq f(\boldsymbol{\sigma} \psi)]
$$

It is not hard to see that for $f: S_{n} \rightarrow\{-1,1\}$, we have

$$
\mathbb{N S}_{\delta}(f)=\frac{1}{2}-\frac{1}{2} \mathbf{E}_{\sigma, \psi \sim N_{\delta}}[f(\boldsymbol{\sigma}) f(\boldsymbol{\sigma} \psi)]=\frac{1}{2}-\frac{1}{2}\left\langle f, T_{\delta} f\right\rangle .
$$

As might be expected, we are aiming to use the Fourier interpretation of noise. The critical part of our definition is that the distribution on $\boldsymbol{\psi}$ is a class distribution; that is, the probability distribution is uniform given a fixed conjugacy class.

Lemma 5.3. Let $U$ be any functional operator operating on functions $f: S_{n} \rightarrow \mathbb{R}$, where $U f$ is such that $U f(\sigma)=\mathbf{E}_{\boldsymbol{\psi}}[f(\boldsymbol{\psi} \sigma)]$ for some random variable $\boldsymbol{\psi}$. If the distribution on $\boldsymbol{\psi}$ is a class distribution, and $\left\{\widehat{f}_{\lambda}\right\}$ is the Fourier spectrum of $f$, then for every $\lambda \vdash n$,

$$
\widehat{U f}{ }_{\lambda}=k_{\lambda} \widehat{f}_{\lambda}
$$

for some constant $k_{\lambda}$ independent of $f$.

Proof. This fact essentially follows from Schur's representation lemma, but we give a proof here for completeness.

Let $\rho$ be any irreducible representation corresponding to the partition $\lambda$. Since $U$ is a linear operator, it suffices to show that for any function $\rho_{i j}$ given by an $(i, j)$-entry of an irreducible representation $\rho$, we have

$$
U \rho_{i j}=k_{\rho} \rho_{i j}
$$

for some constant $k_{\rho}$. Starting from the definition of $U$ and using the fact that $\rho$ is a representation, we get 


$$
\begin{aligned}
\left(U \rho_{i j}\right)(\sigma) & =\mathbf{E}_{\boldsymbol{\psi}}\left[\rho_{i j}(\sigma \boldsymbol{\psi})\right] \\
& =\mathbf{E}_{\boldsymbol{\psi}}\left[(\rho(\sigma) \rho(\boldsymbol{\psi}))_{i j}\right] \\
& =\mathbf{E}_{\boldsymbol{\psi}}\left[\sum_{k=1}^{d_{\rho}} \rho(\sigma)_{i k} \rho(\boldsymbol{\psi})_{k j}\right] \\
& =\sum_{k=1}^{d_{\rho}} \rho(\sigma)_{i k} \mathbf{E}_{\boldsymbol{\psi}}\left[\rho(\boldsymbol{\psi})_{k j}\right] \\
& =\sum_{k=1}^{d_{\rho}} \rho(\sigma)_{i k}\left(\mathbf{E}_{\boldsymbol{\psi}}[\rho(\boldsymbol{\psi})]\right)_{k j} \\
& =\left(\rho(\sigma) \cdot \mathbf{E}_{\boldsymbol{\psi}}[\rho(\boldsymbol{\psi})]\right)_{i j}
\end{aligned}
$$

for any $i$ and $j$. By symmetry and the fact that $\psi$ is a class distribution, we also have

$$
\left(U \rho_{i j}\right)(\sigma)=\mathbf{E}_{\boldsymbol{\psi}}\left[\rho_{i j}(\boldsymbol{\psi} \sigma)=\left(\mathbf{E}_{\boldsymbol{\psi}}[\rho(\boldsymbol{\psi})] \cdot \rho(\sigma)\right)_{i j}\right.
$$

for any $i$ and $j$. Thus, $\rho(\sigma)$ and $\mathbf{E}_{\boldsymbol{\psi}}[\rho(\boldsymbol{\psi})]$ commute for any $\sigma$. Because $\rho$ was taken to be irreducible, the set of matrices $\left\{\rho(\sigma): \sigma \in S_{n}\right\}$ is a spanning set for square matrices of size $d_{\rho}$. It follows that $\mathbf{E}_{\boldsymbol{\psi}}[\rho(\boldsymbol{\psi})]$ commutes with every square matrix of size $d_{\rho}$. This is sufficient to conclude that $\mathbf{E}_{\boldsymbol{\psi}}[\rho(\boldsymbol{\psi})]=k_{\rho} I_{d_{\rho}}$ for some constant $k_{\rho}$, and thus

$$
\left(U \rho_{i j}\right)(\sigma)=\left(\rho(\sigma) \cdot k_{\rho} I_{d_{\rho}}\right)_{i j}=k_{\rho} \rho_{i j}(\sigma) .
$$

The claim is finished by applying the linearity of $U$.

Corollary 5.4. For any positive integer $n$ and $0<\delta<1$ there exist constants $c_{\lambda, \delta}$ such that for every $f: S_{n} \rightarrow\{-1,1\}$, we have

$$
\mathbb{N S}_{\delta}(f)=\sum_{\lambda \vdash n} c_{\lambda, \delta}\left\|\widehat{f}_{\lambda}\right\|^{2} d_{\lambda} \quad \text { and } \quad\left\langle f, T_{\delta} f\right\rangle=\sum_{\lambda \vdash n} c_{\lambda, \delta}^{\prime}\left\|\widehat{f}_{\lambda}\right\|^{2} d_{\lambda}
$$

where $c_{\lambda, \delta}=\frac{1}{2}-\frac{1}{2} c_{\lambda, \delta}^{\prime}$.

Proof. By the previous lemma, we have $\widehat{T_{\delta} f} \lambda=c_{\lambda, \delta}^{\prime} \hat{f}_{\lambda}$ for $\lambda \vdash n$. The second equation follows from applying Plancherel's identity, and the first follows easily from the second.

\section{Low noise sensitivity implies concentration}

In order to show that low noise sensitivity implies some level of concentration, we need to determine the values of the constants $c_{\lambda, \delta}$ in Corollary 5.4. To do this, we consider the theory of characters. Recall that the character $\chi_{\rho}$ of a representation $\rho$ is the function defined by $\chi_{\rho}(\sigma)=\operatorname{tr} \rho(\sigma)$. The character of all representations corresponding to the same partition yield the same character, so $\chi_{\rho}$ is well-defined.

It is not hard to see that representations (characters) of $G$ are also representations (characters) of any subgroup of $G$, although an irreducible representation of $G$ might not be an irreducible representation for every subgroup.

Definition 6.1. For a character $\chi$ of a group $G$ and a subgroup $H \leq G$, we define $\operatorname{Res}_{H} \chi$ to be the character restricted to $H$. When $H$ is clear from context, we simply write Res $\chi$. 
Let us take $W_{k}$ to be the distribution over $S_{n}$ whose samples can be generated the following way: uniformly randomly select $n-k$ of the $n$ points to be guaranteed to be fixed points, and select a random permutation for the other $k$ elements. The noise distribution $N_{\delta}$ (id) is simply $W_{\boldsymbol{k}}$, where $\boldsymbol{k} \sim \operatorname{Binomial}(\delta, n)$ and id is the identity permutation.

Note that

$$
\begin{aligned}
\operatorname{tr} \mathbf{E}_{\boldsymbol{~} \sim W_{k}}[\rho(\boldsymbol{\psi})] & =\mathbf{E}_{\boldsymbol{\psi} \sim W_{k}}[\operatorname{tr} \rho(\boldsymbol{\psi})] \\
& =\mathbf{E}_{\boldsymbol{\psi} \sim W_{k}}\left[\chi_{\rho}(\boldsymbol{\psi})\right] .
\end{aligned}
$$

The character function is a class function on $S_{n}$, so which $n-k$ points are fixed doesn't matter. We will assume that the fixed points are $\{k+1, k+2, \ldots, n\}$. Thus, without loss of generality, we can think of $\boldsymbol{\psi}$ as a uniformly chosen element of $S_{k}$, and thus

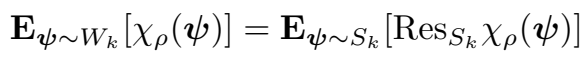

Let $\mu=(k)$. The corresponding representation is the trivial representation (which we will denote triv), whose character is the constant function 1 . Thus,

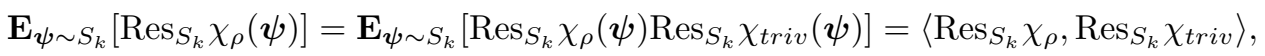

the inner product taken in $S_{k}$. It is known (see, for example, (8.1) in [OO96]) that

$$
\left\langle\operatorname{Res}_{S_{k}} \chi_{\rho}, \operatorname{Res}_{S_{k}} \chi_{t r i v}\right\rangle=\operatorname{dim} \lambda /(k)
$$

where $\lambda$ is the partition corresponding to the irreducible representation $\rho$. It follows that the trace of $\mathbf{E}_{\boldsymbol{\psi} \sim W_{k}}[\rho(\boldsymbol{\psi})]$ is $\operatorname{dim} \lambda /(k)$, and $\mathbf{E}_{\boldsymbol{\psi} \sim W_{k}}[\rho(\boldsymbol{\psi})]=\frac{\operatorname{dim} \lambda /(k)}{\operatorname{dim} \lambda} I_{\operatorname{dim} \lambda}$.

Using the fact that $N_{\delta}$ is a mixture of distributions of the form $W_{k}$, we get:

Proposition 6.2. $c_{\lambda, \delta}^{\prime}=\mathbf{E}_{\boldsymbol{k}}\left[\frac{\operatorname{dim} \lambda /(\boldsymbol{k})}{\operatorname{dim} \lambda}\right]$, where the distribution on $\boldsymbol{k}$ is $\operatorname{Binomial}(n, \delta)$.

We will make use of an expression for $\frac{\operatorname{dim} \lambda /(k)}{\operatorname{dim} \lambda}$ due to Okounkov and Olshanski [OO96]. They actually give a much more general statement:

$$
\frac{\operatorname{dim} \lambda / \mu}{\operatorname{dim} \lambda}=\frac{n !}{(n-k) !} s_{\mu}^{*}(\lambda)
$$

for any partition $\mu \vdash k$. The expression $s_{\mu}^{*}(\lambda)$ is a shifted Schur function. In the same paper [OO96], the authors give a handy interpretation of these shifted Schur functions in terms of tableaux. For our purposes, we only need the case where $\mu=(k)$; in this case, we get

$$
s_{(k)}^{*}\left(x_{1}, x_{2}, \ldots\right)=\sum_{1 \leq i_{1} \leq i_{2} \cdots \leq i_{k}<\infty}\left(x_{i_{1}}-k+1\right)\left(x_{i_{2}}-k+2\right) \cdots x_{i_{k}}
$$

Recall that the dominance ordering on partitions is the partial order such that $\lambda \unrhd \beta$ if $\sum_{i=1}^{k} \lambda_{i} \geq \sum_{i=1}^{k} \beta_{i}$ for all $k \geq 1$. Intuitively, the higher degree representations should contribute more to the noise sensitivity. This is indeed the case. To show this, we prove the following result, which might be of independent interest (we remark that this was proved for $k=2$ in [Dia88]):

Theorem 6.3. Let $\lambda \vdash n$ and $\beta \vdash n$ such that $\lambda \unrhd \beta$. Then

$$
\frac{\operatorname{dim} \lambda /(k)}{\operatorname{dim} \lambda} \geq \frac{\operatorname{dim} \beta /(k)}{\operatorname{dim} \beta}
$$

for any integer $k \geq 0$. 
Proof. It suffices to consider the case that $\lambda \vdash n$ and $\beta \vdash n$ are such that $\lambda \unrhd \beta$ and there does not exist a partition $\alpha \vdash n$ such that $\lambda \unrhd \alpha \unrhd \beta$. In this case, there exist indices $s<t$ such that $\lambda_{s}=\beta_{s}+1, \lambda_{t}=\beta_{t}-1$, and $\lambda_{j}=\beta_{j}$ for $s<j<t$ (this interval might be empty). It suffices to show that $s_{(k)}^{*}\left(\lambda_{1}, \lambda_{2}, \ldots, \lambda_{\ell}\right) \geq$ $s_{(k)}^{*}\left(\beta_{1}, \beta_{2}, \ldots, \beta_{\ell}\right)$.

We prove the statement by induction on $n$ and $k$.

Note that $s_{(k)}^{*}\left(\lambda_{1}, \lambda_{2}, \ldots, \lambda_{\ell}\right)=\left(\lambda_{1}-k+1\right) s_{(k-1)}^{*}\left(\lambda_{1}, \lambda_{2}, \lambda_{3}, \ldots, \lambda_{\ell}\right)+s_{(k)}^{*}\left(\lambda_{2}, \lambda_{3}, \ldots, \lambda_{\ell}\right)$. If $\lambda_{1}=\beta_{1}$, then $\left(\lambda_{2}, \lambda_{3}, \ldots, \lambda_{\ell}\right) \unrhd\left(\beta_{2}, \beta_{3}, \ldots, \beta_{\ell}\right)$, and we are done by induction. Similarly, if $\lambda_{\ell}=\beta_{\ell}$, then $s_{(k)}^{*}(\lambda)=$ $s_{(k-1)}^{*}\left(\lambda_{1}, \lambda_{2}, \ldots, \lambda_{\ell-1}, \lambda_{\ell}\right)\left(\lambda_{\ell}\right)+s_{(k)}^{*}\left(\lambda_{1}, \lambda_{2}, \ldots, \lambda_{\ell-1}\right)$ and we are done by induction. Thus, without loss of generality, we can assume that $s=1$ and $t=\ell$; also, we can assume $\lambda_{1}=\beta_{1}+1, \lambda_{\ell}=\beta_{\ell}-1$, and $\lambda_{2}=\lambda_{3}=\cdots=\lambda_{\ell-1}=\beta_{2}=\beta_{3}=\cdots=\beta_{\ell-1}$.

First, we note that all the terms in the summation are nonnegative. This is not hard to see: in the product, each expression is at most 1 more than the first, and the last expression is positive. Thus, if any negative expression is encountered, one of the expressions will evaluate to zero.

Let $\operatorname{seq}_{k, \ell}$ be the set of nonincreasing sequences $\left\{i_{1}, \ldots, i_{k}\right\}$ where $1 \leq i_{j} \leq \ell$. For a nondecreasing sequence $i=\left\{i_{1}, \ldots, i_{k}\right\}$ of integers in $\{1,2, \ldots, \ell\}$, let $\operatorname{rev}(i)$ be the sequence such that $\operatorname{rev}(i)_{j}=\ell+1-$ $i_{k+1-j}$. For $i \in \operatorname{seq}_{k, \ell}$, we define the function $\pi_{i}: \mathbb{R}^{k} \rightarrow \mathbb{R}$ such that $\pi_{i}(x)=\left(x_{i_{1}}-k+1\right)\left(x_{i_{2}}-k+2\right) \cdots x_{i_{k}}$. By decomposing the sum, it suffices to show that

$$
\pi_{i}(\lambda)+\pi_{\text {rev }(i)}(\lambda) \geq \pi_{i}(\beta)+\pi_{\operatorname{rev}(i)}(\beta)
$$

since summing the equality over all sequences (and halving if $i=\operatorname{rev}(i)$ ) yields the claim.

If $i_{1} \neq 1$ and $i_{k} \neq \ell$, then $\operatorname{rev}(i)_{1} \neq 1$ and $\operatorname{rev}(i)_{k} \neq \ell$, and the claim holds with equality.

If $i_{1}=1$ and $i_{k}=\ell$, then $\operatorname{rev}(i)_{1}=1$ and $\operatorname{rev}(i)_{k}=\ell$ as well. Writing $\bar{i}$ for $\left\{i_{2}, i_{3}, \ldots, i_{k-1}\right.$ and using induction, we have

$$
\begin{gathered}
\pi_{\bar{i}}(\lambda)+\pi_{\operatorname{rev}(\bar{i})}(\lambda) \geq \pi_{\bar{i}}(\beta)+\pi_{\operatorname{rev}(\bar{i})}(\beta) \Longrightarrow \\
\pi_{\bar{i}}(\lambda)+\pi_{\operatorname{rev}(\bar{i})}(\lambda)\left(\beta_{1}+1-k+1\right)\left(\beta_{1}-1\right) \geq \pi_{\bar{i}}(\beta)+\pi_{\operatorname{rev}(\bar{i})}(\beta)\left(\beta_{1}-k+1\right)\left(\beta_{1}\right) \Longrightarrow \\
\pi_{i}(\lambda)+\pi_{\operatorname{rev}(i)}(\lambda) \geq \pi_{i}(\beta)+\pi_{\operatorname{rev}(i)}(\beta)
\end{gathered}
$$

using that $\left(\beta_{1}+1-k+1\right)\left(\beta_{1}-1\right) \geq\left(\beta_{1}-k+1\right)\left(\beta_{1}\right)$ since $k \leq \beta_{1}+1$.

For the remaining cases, suppose without loss of generality that $i_{1}=1$ and $i_{k} \neq \ell$. Then $\operatorname{rev}(i)_{1} \neq 1$ and $\operatorname{rev}(i)_{k}=\ell$. The inequality we need to show is

$$
\left(\beta_{1}-k+2\right)^{(a)}\left(\beta_{1}-k+a+1\right)^{(k-a)}+\left(\beta_{1}-k+1\right)^{(k-a)}\left(\beta_{1}-a\right)^{(a)} \geq 2\left(\beta_{1}-k+1\right)^{(k)}
$$

If $a=0$, the claim is trivial. If $a=k$, the claim follows from the convexity of the rising factorial function for positive arguments.

Suppose $0<a<k$. Then $\left(\beta_{1}-k+2\right)^{(a)}\left(\beta_{1}-k+a+1\right)^{(k-a)}+\left(\beta_{1}-k+1\right)^{(k-a)}\left(\beta_{1}-a\right)^{(a)}$ is

$$
\begin{aligned}
& =\left(\beta_{1}-k+2\right)\left(\beta_{1}-k+3\right)^{(a-1)}\left(\beta_{1}-k+a+1\right)^{(k-a-1)}(\beta)+\left(\beta_{1}-k+1\right)\left(\beta_{1}-k+2\right)^{(k-a-1)}\left(\beta_{1}-a\right)^{(a-1)}\left(\beta_{1}-1\right) \\
& \geq\left(\beta_{1}-k+2\right)\left(\beta_{1}-k+3\right)^{(a-1)}\left(\beta_{1}-k+a+1\right)^{(k-a-1)}(\beta)+\left(\beta_{1}-k\right)\left(\beta_{1}-k+2\right)^{(k-a-1)}\left(\beta_{1}-a\right)^{(a-1)}(\beta) \\
& \geq\left(\beta_{1}-k+1\right)\left(\beta_{1}-k+3\right)^{(a-1)}\left(\beta_{1}-k+a+1\right)^{(k-a-1)}(\beta)+\left(\beta_{1}-k+1\right)\left(\beta_{1}-k+2\right)^{(k-a-1)}\left(\beta_{1}-a\right)^{(a-1)}(\beta) \\
& \geq \beta\left(\beta_{1}-k+1\right)\left[\left(\beta_{1}-k+3\right)^{(a-1)}\left(\beta_{1}-k+a+1\right)^{(k-a-1)}+\left(\beta_{1}-k+2\right)^{(k-a-1)}\left(\beta_{1}-a\right)^{(a-1)}\right] .
\end{aligned}
$$

By induction (with $\beta_{1}+1, k-2$, and $a-1$ for $\beta_{1}, k$, and $a$ respectively), the last expression is greater than $\beta_{1}\left(\beta_{1}-k+1\right) 2\left(\beta_{1}-k+2\right)^{(k-2)}=2\left(\beta_{1}-k+1\right)^{(k)}$, completing the inductive proof. Note that both $a$ and $k-a$ strictly decrease in the inductive step, so after finitely many steps we are in one of the base cases. 
Corollary 6.4. Let $S=\left\{\lambda \vdash n: \lambda_{1} \leq n-\ell\right\}$ for some $\ell$. For every $0 \leq \delta \leq 1$, the maximum of $c_{\lambda, \delta}^{\prime}$ for $\lambda \in S$ occurs when $\lambda=(n-\ell, \ell)$.

Proof. For every $\lambda \in S$, we have $\left.c_{\lambda, \delta}^{\prime}=\mathbf{E}_{[} \frac{\operatorname{dim} \lambda /(\boldsymbol{k})}{\operatorname{dim} \lambda}\right]$. We apply the previous theorem for each $0 \leq k \leq n$, using the fact that $(n-\ell, \ell) \unrhd \lambda$ for all $\lambda \in S$. The result follows by taking the expectation.

This result tells us that as a partition gets more "stretched out", the corresponding representation is more sensitive to noise. We show that $\frac{\operatorname{dim} \lambda /(k)}{\operatorname{dim} \lambda}$ is upper bounded by an expression exponentially decreasing in $k$; we encourage the reader to compare to the hypercube case.

From [Dia88], $\frac{\operatorname{dim} \lambda /(2)}{d_{\lambda}}$ is simple to compute directly:

$$
\frac{\operatorname{dim} \lambda /(2)}{d_{\lambda}}=\frac{\lambda_{1}\left(\lambda_{1}-1\right)+\lambda_{2}\left(\lambda_{1}-1\right)+\lambda_{2}\left(\lambda_{2}-1\right)}{n(n-1)}=\frac{\lambda_{1}-1}{n-1}+\frac{\lambda_{2}\left(\lambda_{2}-1\right)}{n(n-1)}
$$

Let $(a)_{b}$ be the falling factorial function; $(a)_{b}=a(a-1) \cdots(a-b+1)$. It follows from [OO96] that

$$
\frac{\operatorname{dim} \lambda /(k)}{\operatorname{dim} \lambda}=\sum_{i=0}^{k} \frac{\left(\lambda_{1}\right)_{k-i}\left(\lambda_{2}-k+i\right)_{i}}{(n)_{k}}
$$

With this in hand, we show that this quantity is decreasing exponentially in $k$ :

Proposition 6.5. For $\lambda=\left(\lambda_{1}, \lambda_{2}\right) \vdash n$, we have $\frac{\operatorname{dim} \lambda /(k)}{\operatorname{dim} \lambda} \leq\left(\frac{\operatorname{dim} \lambda /(2)}{\operatorname{dim} \lambda}\right)^{k-1}$.

Proof. The case $k=1$ is immediate. When $k \geq 2$, it suffices to show

$$
\frac{\operatorname{dim} \lambda /(k)}{\operatorname{dim} \lambda} \leq \frac{\operatorname{dim} \lambda /(k-1)}{\operatorname{dim} \lambda} \cdot \frac{\operatorname{dim} \lambda /(2)}{\operatorname{dim} \lambda}
$$

It follows from Equation 3 that

$$
\frac{\operatorname{dim} \lambda /(k)}{\operatorname{dim} \lambda}=\frac{\operatorname{dim} \lambda /(k-1)}{\operatorname{dim} \lambda} \frac{\lambda_{1}-k+1}{n-k+1}+\frac{\left(\lambda_{2}\right)_{k}}{(n)_{k}}
$$

Now for $k \geq 2$, we have $\frac{\lambda_{1}-k+1}{n-k+1} \leq \frac{\lambda_{1}-1}{n-1}$. We will show

$$
\frac{\left(\lambda_{2}\right)_{k}}{(n)_{k}} \leq \frac{\operatorname{dim} \lambda /(k-1)}{\operatorname{dim} \lambda} \frac{\lambda_{2}\left(\lambda_{2}-1\right)}{n(n-1)} \quad \text { or equivalently } \quad \frac{\left(\lambda_{2}-2\right)_{k-2}}{(n-2)_{k-2}} \leq \frac{\operatorname{dim} \lambda /(k-1)}{\operatorname{dim} \lambda}
$$

(If $\lambda_{2}<k$, then the left hand side of the first inequality is zero and we are done.) Indeed,

$$
\begin{aligned}
\frac{\left(\lambda_{2}-2\right)_{k-2}}{(n-2)_{k-2}} \leq \frac{\left(\lambda_{2}-1\right)_{k-2}}{(n-1)_{k-2}} & =\frac{\lambda_{1}\left(\lambda_{2}-1\right)_{k-2}}{n(n-1)_{k-2}}+\frac{\lambda_{2}\left(\lambda_{2}-1\right)_{k-2}}{n(n-1)_{k-2}} \\
& \leq \frac{\lambda_{1}\left(\lambda_{1}-1\right)_{k-2}}{(n)_{k-1}}+\frac{\lambda_{2}\left(\lambda_{2}-1\right)_{k-2}}{(n)_{k-1}}=\frac{\left(\lambda_{1}\right)_{k-1}}{(n)_{k-1}}+\frac{\left(\lambda_{2}\right)_{k-1}}{(n)_{k-1}} \leq \frac{\operatorname{dim} \lambda /(k-1)}{\operatorname{dim} \lambda}
\end{aligned}
$$

where the last inequality uses the fact that the left hand side consists of two terms of the summation in Equation 3, and all of the terms in the summation are nonnegative. It follows that 


$$
\begin{aligned}
\frac{\operatorname{dim} \lambda /(k)}{\operatorname{dim} \lambda} & =\frac{\operatorname{dim} \lambda /(k-1)}{\operatorname{dim} \lambda} \frac{\lambda_{1}-k+1}{n-k+1}+\frac{\left(\lambda_{2}\right)_{k}}{(n)_{k}} \\
& \leq \frac{\operatorname{dim} \lambda /(k-1)}{\operatorname{dim} \lambda}\left(\frac{\lambda_{1}-1}{n-1}+\frac{\lambda_{2}\left(\lambda_{2}-1\right)}{n(n-1)}\right) \\
& =\frac{\operatorname{dim} \lambda /(k-1)}{\operatorname{dim} \lambda} \cdot \frac{\operatorname{dim} \lambda /(2)}{\operatorname{dim} \lambda},
\end{aligned}
$$

finishing the proof of the claim.

Corollary 6.6. For a partition $\lambda$ into two parts, $c_{\lambda, \delta}^{\prime} \leq \mathbf{E}\left[\left(\frac{\operatorname{dim} \lambda /(2)}{\operatorname{dim} \lambda}\right)^{\boldsymbol{k}-1}\right]$, where $\boldsymbol{k}$ is distributed as $\operatorname{Binomial}(\mathrm{n}, \delta)$.

We are finally ready to prove our noise sensitivity theorem:

Theorem 6.7. Suppose $f: S_{n} \rightarrow\{-1,1\}$ is such that $\mathbb{N S}_{\delta}(f) \leq \epsilon$ for $\delta>10 / n$. Then $\sum_{\lambda: \lambda_{1}>n-1 / \delta} d_{\lambda}\left\|\widehat{f}_{\lambda}\right\|^{2} \leq$ $\frac{2}{1-2 e^{-0.9}} \epsilon$, and $f$ is $O(\epsilon)$-concentrated to degree $1 / \delta$.

Proof. Recall that

$$
\mathbb{N S}_{\delta}(f)=\frac{1}{2}-\frac{1}{2}\left\langle f, T_{\delta} f\right\rangle=\frac{1}{2}-\frac{1}{2} \sum_{\lambda} c_{\lambda, \delta}^{\prime} d_{\lambda}\left\|\widehat{f}_{\lambda}\right\|^{2} .
$$

Assuming $\mathbb{N S}_{\delta}(f) \leq \epsilon$, we have:

$$
\begin{aligned}
\epsilon & \geq \frac{1}{2}-\frac{1}{2} \sum_{\lambda} c_{\lambda, \delta}^{\prime}\left\|\widehat{f}_{\lambda}\right\|^{2} \\
& =\frac{1}{2}\left(\sum_{\lambda} d_{\lambda}\left\|\widehat{f}_{\lambda}\right\|^{2}-\sum_{\lambda} c_{\lambda, \delta}^{\prime} d_{\lambda}\left\|\widehat{f}_{\lambda}\right\|^{2}\right)=\frac{1}{2}\left(\sum_{\lambda}\left(1-c_{\lambda, \delta}^{\prime}\right) d_{\lambda}\left\|\widehat{f}_{\lambda}\right\|^{2}\right) \geq \frac{1}{2}\left(\sum_{\lambda: \lambda_{1} \leq n-1 / \delta}\left(1-c_{\lambda, \delta}^{\prime}\right) d_{\lambda}\left\|\widehat{f}_{\lambda}\right\|^{2}\right) .
\end{aligned}
$$

We used Parseval's identity in the first equality, which says that $\sum_{\lambda} d_{\lambda}\left\|\widehat{f}_{\lambda}\right\|^{2}=1$ for a Boolean valued function.

We maximize $c_{\lambda, \delta}^{\prime}$ over $\lambda \vdash n$ where $\lambda_{1} \leq n-1 / \delta$. By Corollary 6.4, the maximum will occur at $\lambda=(n-1 / \delta, 1 / \delta)$. By Corollary 6.6, we have $c_{\lambda, \delta}^{\prime} \leq \mathbf{E}\left[\left(\frac{\operatorname{dim} \lambda /(2)}{\operatorname{dim} \lambda}\right)^{\boldsymbol{k}-1}\right]$. Using the moment generating function for the binomial distribution, we have $c_{\lambda, \delta}^{\prime} \leq \frac{\operatorname{dim} \lambda}{\operatorname{dim} \lambda /(2)}\left(1-\delta\left(1-\frac{\operatorname{dim} \lambda /(2)}{\operatorname{dim} \lambda}\right)\right)^{n}$.

We analyze the quantity $1-\frac{\operatorname{dim} \lambda /(2)}{\operatorname{dim} \lambda}$ using simple algebra and the fact that $\lambda_{1}+\lambda_{2}=n$ :

$$
\begin{aligned}
1-\frac{\operatorname{dim} \lambda /(2)}{d_{\lambda}} & =\frac{n(n-1)-\lambda_{1}\left(\lambda_{1}-1\right)-\lambda_{2}\left(\lambda_{1}-1\right)-\lambda_{2}\left(\lambda_{2}-1\right)}{n(n-1)} \\
& =\frac{n(n-1)-n\left(\lambda_{1}-1\right)-\lambda_{2}\left(\lambda_{2}-1\right)}{n(n-1)} \\
& =\frac{n \lambda_{2}-\lambda_{2}\left(\lambda_{2}-1\right)}{n(n-1)} \\
& =\frac{\lambda_{2}\left(\lambda_{1}+1\right)}{n(n-1)}
\end{aligned}
$$


Assume $\delta>10 / n$. Then for $\lambda=(n-1 / \delta, 1 / \delta)$ we have $1-\frac{\operatorname{dim} \lambda /(2)}{\operatorname{dim} \lambda} \geq 0.9 /(\delta n)$ and thus $\frac{\operatorname{dim} \lambda}{\operatorname{dim} \lambda /(2)} \leq$ $\frac{1}{1-0.9 /(\delta n)} \leq 2$, so $c_{\lambda, \delta}^{\prime} \leq 2(1-\delta(0.9 /(\delta n)))^{n} \leq 2 e^{-0.9}$. It follows that

$$
\frac{1}{2}\left(\sum_{\lambda: \lambda_{1} \leq n-1 / \delta}\left(1-c_{\lambda, \delta}^{\prime}\right) d_{\lambda}\left\|\widehat{f}_{\lambda}\right\|^{2}\right) \geq \frac{1}{2}\left(\sum_{\lambda: \lambda_{1} \leq n-1 / \delta}\left(1-2 e^{-0.9}\right) d_{\lambda}\left\|\widehat{f}_{\lambda}\right\|^{2}\right)
$$

and therefore $\sum_{\lambda: \lambda_{1} \leq n-1 / \delta} d_{\lambda}\left\|\widehat{f}_{\lambda}\right\|^{2} \leq \frac{2}{1-2 e^{-0.9}} \epsilon$.

To show that this implies $O(\epsilon)$-concentration on functions of order at most $1 / \delta$, we take $p(\sigma)$ to be the function such that $\widehat{p}_{\lambda}=\widehat{f}_{\lambda}$ if $\lambda_{1}>n-1 / \delta$ and $\widehat{p}_{\lambda}=0$ otherwise. It follows that

$$
\mathbf{E}_{\boldsymbol{\sigma}}\left[(p(\boldsymbol{\sigma})-f(\boldsymbol{\sigma}))^{2}\right]=\sum_{\lambda: \lambda_{1}>n-1 / \delta} d_{\lambda}\left\|\widehat{f}_{\lambda}\right\|^{2} \leq \frac{1}{1-2 e^{-0.9}} \epsilon .
$$

The fact that $p$ is a function of degree at most $1 / \delta$ follows from the support of the Fourier spectrum. We remark that the assumption that $\delta>10 / n$ can be removed if one replaces degree $1 / \delta$ with $10 / \delta$, since this modified statement becomes trivial if $\delta \leq 10 / n$.

\section{$7 \quad$ Linear threshold functions}

Recall the definition of linear threshold functions over $S_{n}$.

Definition 7.1. We say that a function $f: S_{n} \rightarrow\{-1,1\}$ is a linear threshold function if there exist weights $w_{i j}$ for $1 \leq i \neq j \leq n$ and a threshold $\theta$ such that $f(\sigma)=\operatorname{sgn}\left(\sum_{i, j} w_{i j} \mathbf{1}[\sigma(i)=j]-\theta\right)$ for all $\sigma \in S_{n}$.

We remind the reader of Peres' Theorem in the Boolean hypercube case:

Theorem 7.2. Let $f:\{0,1\}^{n} \rightarrow\{-1,1\}$ be a linear threshold function. Then $\mathbb{N S}_{\delta}(f)=O(\sqrt{\delta})$ under the uniform distribution on $\{0,1\}^{n}$.

The notion of noise sensitivity in a product distribution is much easier to deal with, because each coordinate can be handled independently. Similar to the argument in [BOW10], we will (nontrivially) reduce to the uniform distribution on the Boolean hypercube case. We give a definition of noise sensitivity here that is equivalent to the definition used already.

Definition 7.3. For $f: S_{n} \rightarrow\{-1,1\}$, we can define $\mathbb{N S}_{\delta}(f)$ as follows: Let $\boldsymbol{\alpha} \sim \operatorname{Binomial}(n, \delta)$, and choose a uniformly random subset $\boldsymbol{S}$ of $[n]$ of cardinality $\boldsymbol{\alpha}$. Let $\boldsymbol{\psi}$ be a uniformly random permutation in $S_{n}$ such that every element of $[n] \backslash \boldsymbol{S}$ is a fixed point of $\boldsymbol{\psi}$. Then

$$
\mathbb{N S}_{\delta}(f)=\underset{\boldsymbol{\sigma}, \boldsymbol{\psi}}{\operatorname{Pr}}[f(\boldsymbol{\sigma}) \neq f(\boldsymbol{\psi} \boldsymbol{\sigma})]
$$

where $\boldsymbol{\sigma}$ is chosen uniformly at random from $S_{n}$.

It is not hard to see that this definition is consistent with our previous definitions.

Theorem 7.4 (Theorem 3.5). Let $f: S_{n} \rightarrow\{-1,1\}$ be a linear threshold function. Then $\mathbb{N S}_{\delta}(f) \leq 2 \sqrt{\delta}$.

Proof. Our proof will closely mirror the proof of Peres' Theorem given in [O'D07]. Analogously to the proof there, we will prove a slightly stronger statement. Let $F_{1}, \ldots, F_{m}$ be any partition of $n$. For each $F_{i}$, let $\psi_{i}$ be an arbitrary permutation such that every point of $[n] \backslash F_{i}$ is a fixed point of $\psi_{i}$. Our strategy is to analyze the experiment where we apply one of the $\psi_{i}$ 's to $\sigma$ at random. 
Lemma 7.5. Let $f: S_{n} \rightarrow\{-1,1\}$ be a linear threshold function. Let $F_{1}, \ldots, F_{m} \subseteq[n]$ be a partition of $[n]$ and let $\psi_{1}, \ldots, \psi_{m}$ be permutations as previously mentioned. Then $\operatorname{Pr}_{\boldsymbol{\sigma}, i}\left[f(\boldsymbol{\sigma}) \neq f\left(\psi_{\boldsymbol{i}} \boldsymbol{\sigma}\right)\right] \leq m^{-1 / 2}$.

When $m=1 / \delta$, it is straightforward to check that the distribution on $\psi_{i} \sigma$ is the same as our previously mentioned noise experiment, when the partition and the $\psi_{i}$ 's are chosen uniformly at random as well. Every coordinate in $\sigma$ is non-fixed with probability $\delta$.

Proof. We note that all the $\psi_{i}$ 's commute under composition. We identify every string $x \in\{0,1\}^{m}$ with the permutation $\psi_{I_{1}(x)} \boldsymbol{\sigma}$, where $\psi_{I_{1}(x)}$ denotes the composition of the $\psi_{i}$ 's where $x_{i}=1$. We note that $\psi_{I_{1}(x)} \boldsymbol{\sigma}$ is uniformly distributed on $S_{n}$ if $\boldsymbol{\sigma}$ is. We define $g_{\sigma}:\{0,1\}^{m} \rightarrow\{-1,1\}$ such that $g(x)=f\left(\psi_{I_{1}(x)} \sigma\right)$. We have

$$
\begin{array}{rrr}
\operatorname{Pr}_{\boldsymbol{\sigma}, i}\left[f(\boldsymbol{\sigma}) \neq f\left(\psi_{\boldsymbol{i}} \boldsymbol{\sigma}\right)\right] & = & \mathbf{E}_{\boldsymbol{\sigma}}\left[\operatorname{Pr}_{\boldsymbol{i}}\left[f(\boldsymbol{\sigma}) \neq f\left(\psi_{\boldsymbol{i}} \boldsymbol{\sigma}\right)\right]\right] \\
& =\mathbf{E}_{\boldsymbol{\sigma}}\left[\boldsymbol{P r}_{\boldsymbol{x}, \boldsymbol{i}}\left[f\left(\psi_{I_{1}(\boldsymbol{x})} \boldsymbol{\sigma}\right) \neq f\left(\psi_{I_{1}\left(\boldsymbol{x}^{(i)}\right)} \boldsymbol{\sigma}\right)\right]\right]=\mathbf{E}_{\boldsymbol{\sigma}}\left[\mathbf{P r}_{\boldsymbol{x}, i}\left[g_{\boldsymbol{\sigma}}(\boldsymbol{x}) \neq g_{\boldsymbol{\sigma}}\left(\boldsymbol{x}^{(i)}\right)\right],\right.
\end{array}
$$

where we have used $\boldsymbol{x}^{(i)}$ to denote $\boldsymbol{x}$ with the $\boldsymbol{i}$ th bit flipped.

Consider $\operatorname{Pr}_{\boldsymbol{x}, \boldsymbol{i}}\left[g_{\sigma}(\boldsymbol{x}) \neq g_{\sigma}\left(\boldsymbol{x}^{(\boldsymbol{i})}\right)\right]$, the quantity inside the expectation with $\boldsymbol{\sigma}$ fixed to $\sigma$. Because $f$ is a linear threshold function, $g$ is also a linear threshold function in the traditional binary sense. Further, this probability is precisely the average influence of $g$. It is well-known that if $g$ is a linear threshold function, the average influence is at most $m^{-1 / 2}$. Thus, the quantity inside the above expectation is at most $m^{-1 / 2}$, so the expectation is at most $m^{-1 / 2}$, finishing the claim.

To complete the proof of the Theorem, we notice that the noise experiment can be thought of in the following way, when $\delta=1 / m$ for an integer $m$ : For each $i \in[n]$, put $i$ in one of $\boldsymbol{F}_{1}, \ldots, \boldsymbol{F}_{m}$ uniformly at random. Every coordinate is in any $\boldsymbol{F}_{j}$ with probability $\delta=1 / \mathrm{m}$. We randomly choose permutations $\boldsymbol{\psi}_{j}$ where $j \in[m]$ such that every point in $[n] \backslash \boldsymbol{F}_{j}$ is fixed by $\boldsymbol{\psi}_{j}$.

Using the lemma:

$$
\mathbb{N S}_{\delta}(f)=\mathbf{E}_{\boldsymbol{F}_{1}, \ldots, \boldsymbol{F}_{m}, \boldsymbol{\psi}_{1}, \ldots, \boldsymbol{\psi}_{m}}\left[\operatorname{Pr}_{\boldsymbol{\sigma}, \boldsymbol{i}}\left[f(\boldsymbol{\sigma}) \neq f\left(\boldsymbol{\psi}_{\boldsymbol{i}} \boldsymbol{\sigma}\right)\right]\right] \leq m^{-1 / 2}=\sqrt{\delta}
$$

If $\delta$ is not the reciprocal of an integer, we use the fact that noise sensitivity is increasing in $\delta$, and round up to the nearest reciprocal of an integer, which can not cause us to pay more than factor of 2 , increasing our bound to $2 \sqrt{\delta}$.

\section{Applications to learning}

In this section we prove our main theorems which apply to learning.

Theorem 8.1. Let $\mathcal{C}$ be the class of linear threshold functions over $S_{n}$. There is an algorithm that agnostically learns $\mathcal{C}$ under the uniform distribution on $\mathcal{D}$, using $n^{O\left(1 / \epsilon^{4}\right)}$ time and examples.

Proof. Combining Theorem 6.7 and Theorem 7.4 (Theorem 3.5), we get that $\mathcal{C}$ is $\epsilon^{2}$-concentrated up to degree $O\left(1 / \epsilon^{4}\right)$. It follows that the algorithm of [KKMS08] agnostically learns $\mathcal{C}$ under the uniform distribution on $S_{n}$ using $n^{O\left(1 / \epsilon^{4}\right)}$ time and examples.

Theorem 8.2 (Theorem 3.2). Let $\mathcal{C}$ be the class of functions of $k$ linear threshold functions over $S_{n}$. There is an algorithm that agnostically learns $\mathcal{C}$ under the uniform distribution on $\mathcal{D}$, using $n^{O\left(k^{2} / \epsilon^{4}\right)}$ time and examples. 
Proof. Since $\mathbb{N S}_{\delta}(f)$ is a probability, by the union bound $\mathbb{N S}_{\delta}\left(h\left(f_{1}, f_{2}, \ldots, f_{k}\right)\right) \leq \sum_{i=1}^{k} \mathbb{N S}_{\delta}\left(f_{i}\right)$. If each $f_{i}$ is a linear threshold function, we set $\delta=k^{2} / \epsilon^{4}$ so that $\mathbb{N S}_{\delta}\left(f_{i}\right) \leq \epsilon^{2} / k$ for each $i$, and $\mathbb{N S}_{\delta}\left(h\left(f_{1}, f_{2}, \ldots, f_{k}\right)\right) \leq$ $\epsilon^{2}$. The result follows similarly to the previous proof.

As in our proof of our noise sensitivity bound, for a string $x \in\{0,1\}^{n}$ we define $I_{1}(x)$ as the set of indices where $x_{i}=1$. Define $I_{0}(x)$ similarly. Define $\mathcal{U}_{m}$ to be the uniform distribution over strings where $\left|I_{1}(x)\right|=m$.

Theorem 8.3. There is an algorithm running in time $n^{O\left(1 / \epsilon^{4}\right)}$ for agnostically learning with respect to the class of halfspaces over $\{0,1\}^{m}$ under the distribution $\mathcal{U}_{m}$.

Proof. Let $f^{*}(x)=\operatorname{sgn}\left(\sum_{i} w_{i} x_{i}\right)$ be the most accurate linear threshold function in computing the target function $t$. From the previous section, we know $\mathbb{N S}_{\epsilon^{4}}\left(f^{*}(x)\right) \leq O\left(\epsilon^{2}\right)$, so $f^{*}$ is $O\left(\epsilon^{2}\right)$-concentrated to degree $1 / \epsilon^{4}$.

Convert every example $\langle x, f(x)\rangle$ where $x$ is drawn from $\mathcal{U}_{m}$ to a permutation $\sigma$ by uniformly randomly assigning a random permutation from $I_{1}(x) \rightarrow[k]$ and $I_{0}(x) \rightarrow[n] \backslash[k]$. Note that there exists a linear threshold function over permutations that classifies at least as well as $f^{*}$, since the classifier

$$
g(\sigma)=\operatorname{sgn}\left(\sum_{i} w_{i} \mathbf{1}[\sigma(i) \in[k]]\right)
$$

is a linear threshold function, and is consistent with $f^{*}$ over strings in $\mathcal{U}_{m}$. Further, every permutation is equally likely, so the resulting marginal distribution of the first component of the examples under the transformation is the uniform distribution over $S_{n}$. Therefore, the SVM algorithm described earlier will output a hypothesis with error at most $\epsilon$ worse than the error of $g$. To classify future instances, we can convert from a bit string to a permutation in the same way.

Theorem 8.4. There is an algorithm running in time $n^{O\left(k^{2} / \epsilon^{4}\right)}$ for agnostically learning with respect to the class of functions of $k$ halfspaces over $\{0,1\}^{n}$ under the distribution $\mathcal{U}_{m}$.

Proof. Since $\mathbb{N S}_{\delta}(f)$ is a probability, by the union bound $\mathbb{N S}_{\delta}\left(h\left(f_{1}, f_{2}, \ldots, f_{k}\right)\right) \leq \sum_{i=1}^{k} \mathbb{N S}_{\delta}\left(f_{i}\right)$. If each $f_{i}$ is a linear threshold function, we set $\delta=k^{2} / \epsilon^{4}$ so that $\mathbb{N S}_{\delta}\left(f_{i}\right) \leq \epsilon^{2} / k$ for each $i$, and $\mathbb{N S}_{\delta}\left(h\left(f_{1}, f_{2}, \ldots, f_{k}\right)\right) \leq$ $\epsilon^{2}$. The result follows similarly to the previous proof.

One way of viewing the above theorem is that the algorithm learns under the uniform distribution over all permutations of the string $1^{m} 0^{n-m}$. This reduction can be applied to learn over all permutations of any $n$-character string over any alphabet. The symmetric group case is the case where all the characters in the string are distinct; we take $X=[n]$, and the distribution is uniform over all permutations of $(1,2, \ldots, n)$.

We can apply our theorems to learning over permutation invariant distributions.

Theorem 8.5 (Theorem 3.1). Let $\mathcal{D}$ be any permutation invariant distribution over $\{0,1\}^{n}$. There is an algorithm running in time $n^{O\left(k^{2} / \epsilon^{4}\right)}$ for agnostically learning with respsect to the class of functions of $k$ halfspaces over $\{0,1\}^{n}$ under the distribution $\mathcal{D}$.

Proof. We partition the examples into $n+1$ bins depending on the number of 1 's in the example. Thus, the problem is reduced to learning over $\mathcal{U}_{0}, \mathcal{U}_{1}, \ldots, \mathcal{U}_{n}$. We then run the learning algorithm for each bin once enough examples are seen. We note that we might have to account for some loss due to an insufficient number of examples in some bin, but this can be factored into the $\epsilon$; details can be found in [BOW10].

To extend this to distributions over $\{1,2, \ldots, B\}^{n}$, we first define linear threshold functions for this domain. This definition is consistent with the definition we made earlier. 
Definition 8.6. We say that $f:\{1,2, \ldots, B\}^{n} \rightarrow\{-1,1\}$ is a linear threshold function if $f$ can be expressed as

$$
f(x)=\operatorname{sgn}\left(w_{i j} \mathbf{1}\left[x_{i}=j\right]\right)
$$

for some scalars $w_{i j}$ with $1 \leq i \leq n$ and $1 \leq j \leq B$.

Theorem 8.7 (Theorem 3.4). Let $\mathcal{D}$ be any permutation invariant distribution over $\{1,2, \ldots, B\}^{n}$ for constant $B$. There is an algorithm running in time $n^{O\left(k^{2} / \epsilon^{4}+B\right)}$ for agnostically learning with respect to the class of functions of $k$ linear threshold functions over $\{0,1\}^{n}$ under the distribution $\mathcal{D}$.

Proof. The proof is virtually the same as the proof of Theorem 3.1, except that the number of bins is now $\left(\begin{array}{c}n+B-1 \\ B-1\end{array}\right)=\operatorname{poly}\left(n^{B}\right)$, via a "stars and bars" counting argument.

We remark that our proof of bounded noise sensitivity of linear threshold functions over the symmetric group is robust to other function classes. The property of linear threshold functions we used is that the class of "Booleanized" versions of linear threshold functions over $S_{n}$ are linear threshold functions over $\{0,1\}^{n}$, which have bounded total influence. In fact, our proof goes through for other function classes as long as the class is closed under negating and fixing variables and the resulting Boolean functions have bounded average influence. It is also known that in the Boolean domain, a bound on noise sensitivity implies a bound on average influence $\left[\mathrm{DHK}^{+} 10\right]$, and further, any $\epsilon$-concentration result on functions of up to order $d$ implies a bound on noise sensitivity [BOW10]. So we can derive positive learning results for noise sensitivity under the symmetric group given $\epsilon$-concentration results over the Boolean cube.

For example, we can define a size-s, depth- $c$ circuit over $X^{n}$ to be a circuit of size $s$ and depth $c$ computing a function $f:\{0,1\}^{n \cdot|X|} \rightarrow\{-1,1\}$, whose inputs are the $0 / 1$ indicator variables corresponding to events of the form $X_{i}=j$. Similarly, a size- $s$, depth- $c$ circuit over $S_{n}$ is a circuit of size $s$ and depth $c$ consistent with a function $\{0,1\}^{n^{2}} \rightarrow\{-1,1\}$ whose inputs are the $0 / 1$ indicator variables corresponding to events of the form $\sigma(i)=j$ for $1 \leq i, j \leq n$.

With respect to the uniform distribution on $\{0,1\}^{n}$, [Bop97] proved the following:

Theorem 8.8. The average influence of a function $f:\{0,1\}^{n} \rightarrow\{-1,1\}$ computable by a size $s$, depth $c$ circuit over $\{0,1\}^{n}$ is $O\left(\log ^{c-1} s\right) / n$.

Specifically, it is not hard to modify our noise sensitivity proof to show that $\mathbb{N S}_{\delta}(f) \leq O\left(\delta \log ^{c-1} s\right)$ for any function $f: S_{n} \rightarrow\{-1,1\}$ computable by a size $s$, depth $c$ circuit. Thus, we get results of the following flavor:

Theorem 8.9. Let $\mathcal{D}$ be any permutation invariant distribution over $\{1,2, \ldots, B\}^{n}$ for constant $B$. There is an algorithm running in time $n^{O\left(\log ^{c-1} s / \epsilon^{2}\right)+B}$ for agnostically learning with respect to the class of size $s$, depth c circuits under the distribution $\mathcal{D}$.

\section{References}

[Bon95] Dan Boneh. Learning using group representations. In Proceedings of the eighth annual conference on Computational learning theory, pages 418-426. ACM, 1995.

[Bop97] Ravi B Boppana. The average sensitivity of bounded-depth circuits. Information Processing Letters, 63(5):257-261, 1997.

[BOW10] Eric Blais, Ryan O'Donnell, and Karl Wimmer. Polynomial regression under arbitrary product distributions. Machine learning, 80(2):273-294, 2010. 
[DHK ${ }^{+}$10] Ilias Diakonikolas, Prahladh Harsha, Adam Klivans, Raghu Meka, Prasad Raghavendra, Rocco A Servedio, and Li-Yang Tan. Bounding the average sensitivity and noise sensitivity of polynomial threshold functions. In Proceedings of the 42nd ACM symposium on Theory of computing, pages 533-542. ACM, 2010.

[Dia88] Persi Diaconis. Group representations in probability and statistics. Lecture Notes-Monograph Series, 11:i-192, 1988.

[Ful97] William Fulton. Young tableaux: with applications to representation theory and geometry, volume 35. Cambridge University Press, 1997.

[HGG09] Jonathan Huang, Carlos Guestrin, and Leonidas Guibas. Fourier theoretic probabilistic inference over permutations. The Journal of Machine Learning Research, 10:997-1070, 2009.

[KB08] Risi Kondor and Karsten M Borgwardt. The skew spectrum of graphs. In Proceedings of the 25th international conference on Machine learning, pages 496-503. ACM, 2008.

[KHJ07] Risi Kondor, Andrew Howard, and Tony Jebara. Multi-object tracking with representations of the symmetric group. In International Conference on Artificial Intelligence and Statistics, pages 211-218, 2007.

[KKMS08] Adam Tauman Kalai, Adam R Klivans, Yishay Mansour, and Rocco A Servedio. Agnostically learning halfspaces. SIAM Journal on Computing, 37(6):1777-1805, 2008.

[KOS04] Adam R. Klivans, Ryan O'Donnell, and Rocco A. Servedio. Learning intersections and thresholds of halfspaces. J. Comput. Syst. Sci., 68(4):808-840, 2004.

[KSS94] Michael J Kearns, Robert E Schapire, and Linda M Sellie. Toward efficient agnostic learning. Machine Learning, 17(2-3):115-141, 1994.

[KST09] Adam Tauman Kalai, Alex Samorodnitsky, and Shang-Hua Teng. Learning and smoothed analysis. In Foundations of Computer Science, 2009. FOCS'09. 50th Annual IEEE Symposium on, pages 395-404. IEEE, 2009.

[LMN93] Nathan Linial, Yishay Mansour, and Noam Nisan. Constant depth circuits, fourier transform, and learnability. Journal of the ACM (JACM), 40(3):607-620, 1993.

[O’D07] Ryan O'Donnell. Lecture notes on analysis of boolean functions. 2007.

[OO96] Andrei Okounkov and Grigori Olshanski. Shifted schur functions, 1996.

[OW09] Ryan O'Donnell and Karl Wimmer. Kkl, kruskal-katona, and monotone nets. In Foundations of Computer Science, 2009. FOCS'09. 50th Annual IEEE Symposium on, pages 725-734. IEEE, 2009 .

[Per04] Yuval Peres. Noise stability of weighted majority. arXiv preprint math/0412377, 2004.

[SJ06] Pannagadatta K Shivaswamy and Tony Jebara. Permutation invariant svms. In Proceedings of the 23rd international conference on Machine learning, pages 817-824. ACM, 2006. 